\title{
Harmonic Source Identification of a Distributed Generator, and Compensation of the Voltage Change Caused by Changing Generation
}

\author{
Bálint Hartmann ${ }^{1}$ and Supervisor Author András Dán, Dr. ${ }^{2}$ \\ ${ }^{1}$ Graduate student at the Department of Electric Power Engineering \\ Budapest University of Technology and Economics \\ Budapest (Hungary) \\ E-mail: Hartmann.balint@gmail.com \\ ${ }^{2}$ Professor at the Department of Electric Power Engineering \\ Budapest University of Technology and Economics \\ Budapest (Hungary) \\ Phone: +36 1463 3019, e-mail: dan.andras@vet.bme.hu
}

\begin{abstract}
Energy industry is facing one of the biggest transition of its history. The decreasing amount of fossil fuels, the reduction of emissions, the rising energy prices all advert renewable energy resources. The penetration of distributed generation has already begun, but the integration into the old network structure is still in its infancy; the technological change is not yet followed by the needful aspect change. For this reason, extended investigation of the connection terms is indispensable.

In the present paper two aspects are examined. First, a computer model is created, and the verification process is done, using the method of harmonic source identification. The second part of the paper focuses on the steady voltage change caused by the connection of the generator. After simulations, the authors set a potential course to solve the problem, using only the built-in power electronic devices.
\end{abstract}

\section{Keywords}

Computer simulation, power quality, distributed generation, harmonic source identification, reactive power compensation

\section{Introduction}

Effects on line power caused by distributed generation can be very multiple - emission of harmonics, flicker, and the steady voltage change as the result of the connection all takes effect. The standards, that are in force nowadays, regulate the limit values for the majority of such measurable parameters, but contain nothing about control problems. In turn, a distributed generator deemed to have negative effects in a particular case might have positive ones under conditions. Modern power electronic converters are available at most grid connection points, but their role is restricted to inject the generated energy into the grid. The challenge of the future is to find the proper ways to take them into the control process.
The subject of the paper is an $800 \mathrm{~kW}$ wind generator, mounted in the periphery of the Hungarian village, Mecsér. The built-in power and the scheme of the grid connection characterize the Hungarian wind generators well. Based on the available measurements, a computer model was made for DIgSILENT PowerFactory, the paper contains the verification process. The root of the work is the harmonic source identification, which is often used in engineering; this method of measurement techniques is reviewed in detail in [1]. The computer model made can be used for further investigations.

The other objective of the paper is the examination of a control problem. The connection or the disconnection of a generator causes significant steady voltage change at the grid connection point. The current injected by the distributed generator is purely active, so the voltage change may be bigger than the permitted (2-3\% commonly), depending for example on the momentary power generation or the state of the network. To avoid this phenomenon, the generator can be taken into the local voltage control process, using its power electronic devices. There is no generally accepted or recommended procedure to manage this situation, thus the aim of the paper is to set a potential course.

\section{A. Technical backgorund}

Wind generators are symbols of the renewable energy movement. Their number and capacity is steadily increasing all over the world, and in Hungary as well. But what price do we have to pay for the relatively low production price? Flicker, harmonic distortion, voltage fall or rise in connection with the changing generation can all occur. The aim of an engineer is to determine how big is their effect. For the examination, the newest wind generator of Hungary's only 63 MW capacity was chosen, the $800 \mathrm{~kW}$ unit mounted near Mecsér. The generator is the E-48 type of Enercon $\mathrm{GmbH}$, a wellknown one in Hungary, because four pieces were already in operation before. In the base of the tower stands the $0,4 / 22 \mathrm{kV}$ Pauwels transformer with the apparent power 
of $900 \mathrm{kVA}$. The generator delivers power into the network through three parallel power electronic converters, products of Enercon. The inverter is working with pulse width modulation. The generator is connected to a MV feeder, that ends in the $120 / 20 \mathrm{kV}$ substation of Horvátkimle.

The model made with DIgSILENT PowerFactory contains some simplifications, for example no public consumers were built into the model, and the changing cross section size of the feeder was ignored. To sum all up, the wind generator of Mecsér is a good average of the Hungarian wind generators speaking either about the size or the connection scheme - an ideal one to investigate.

\section{The Harmonic Source Identification}

It is often said in daily life, that the best defence is prevention. In the present case it means, that we have to find the harmonic sources connected to the grid, determine the size of the currents and the share of each consumer in the voltage distortion. In absence of knowledge we could be disposed to believe, the generator in the highest degree causes the voltage distortion measured at the connection point.

The power electronics of today convert the energy with the aim to reach the best efficiency. This usually results that the current consumed by the non-linear consumers connected to the grid differs from the $50 \mathrm{~Hz}$ fundamental frequency and contains harmonics. This current flowing through the network impedance creates a voltage drop, distorting the sinusoidal signal. Though based upon this phenomenon non-linear consumers are treated as current sources, it cannot be stated that the highest value of harmonic voltage drop occurs at the connection point. Because the relating standard contains limits for the voltage distortion, the injected harmonic current and the measurement-point impedance of the grid must be known; but this would be easy only when dealing with one consumer - and this rarely happens in practice. Another problems may show up because of the vector nature of the voltage distortions. It is not sure at all, that a new non-linear consumer will raise the amount of distortion.

To sum up all that has been mentioned, daily practice requires such a method for identification, that is capable of determining the standardised value for the voltage distortion of the measured feeder based on the regulations of Hungarian Standard MSZ EN 50160, capable of identifying not only dominant sources, and does not encroach into the technology of the consumer. The method of monoparameter variation suits all these aspects. It is based on the fact, that though the energy system is in constant change, it is always possible to find two, in time following measurements, where only one pair of parameters changed in respect of the analysed quantum. This method is fully discussed in [1].

\section{A. Analysing the Harmonic Currents}

Two measurements were available for the authors for the identification process. A short non-delay measurement with averaging at every $160 \mathrm{~ms}$, and a 10 day long measurement with averaging every 10 minutes. From these, the non-delay measurement would be ideal for the method of monoparameter variation, but it did not match certain criteria; the variation of the harmonic currents happened usually with low speed and low amplitude, interfering to use it for further analysis. Though the 10 day long measurement can tell us much about the behaviour of the generator, of course only a more imprecise identification could be made.

These 10 minute averages show different ways to cope with. For example correlation of the fundamental frequency current and harmonic current of the generator can be computed. These characteristics look very similar for every harmonic, and show almost no relationship between the measured currents. In many cases effect of public consumers can be noticed (during the evening hours amount of the fifth and seventh harmonics increase). Other way to examine the measurement is to compare the harmonic current of the generator and the harmonic voltage distortion measured at the grid connection point.

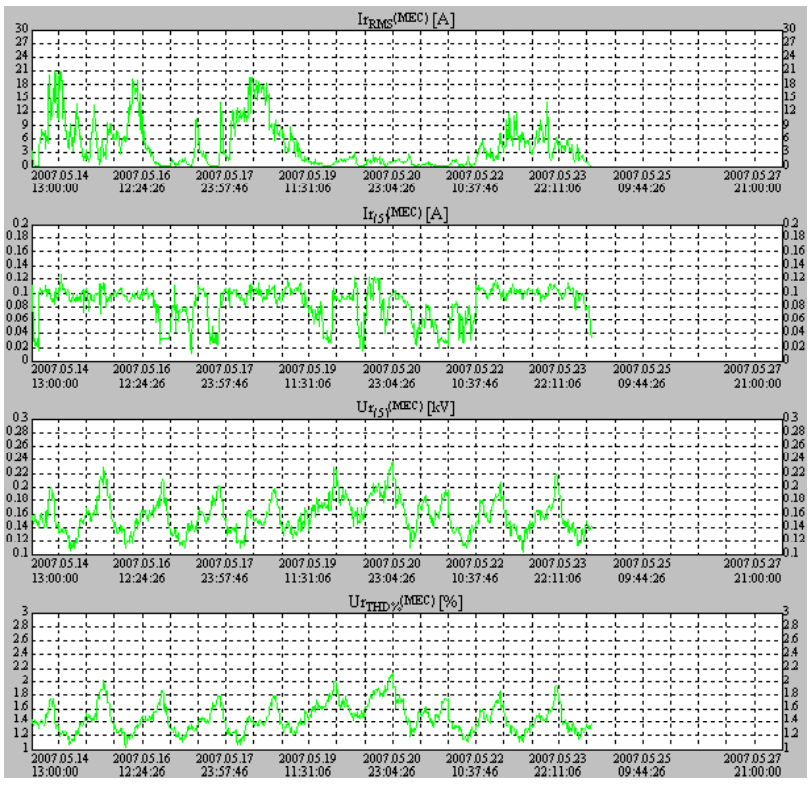

Fig. 1. Time display of the 10 day long measurement

Figure 1. shows a good example. The four curves are the RMS current of the generator, the fifth harmonic current of the generator, the fifth harmonic voltage drop at the connection point, and the total harmonic distortion for the same point. As the figure indicates, there is no relationship in practice between the harmonic current of the generator and the harmonic voltage distortion. Furthermore, the harmonic injection of the generator has very little effect on the harmonic voltage of the connection point. Another representative relationship is likely. If we compose the sequence components of a certain harmonic current, it can be stated, how big is the connection between it and the other consumers connecting to the grid. The characteristics show very similar results again; in most cases the dominant 
sequence component is the one that is generated by the grid, so the zero sequence component for the third harmonic, the negative sequence component for the fifth harmonic, the positive sequence component for the seventh harmonic and so on. This lets to draw the conclusion that the majority of harmonic currents are getting into the measurement circuit from the communal grid. The method of this phenomenon namely how these currents get in needs further investigation, and the better knowledge of the power electronic converter is necessary.

The authors conclude, that the measurement is heavily loaded with background noise, and the 10 minute averages make the identification more imprecise. Despite these, it is stated, that the distributed generator is not a dominant harmonic source, the distortion caused by it does not change the network signal much.

\section{B. Analysing the Harmonic Voltage Distortion}

Going on with voltage characteristics it must be pointed out, that the Hungarian Standard MSZ EN 50160 contains regulation for only harmonic voltages and not for harmonic currents. It includes the distortion limits for certain harmonic voltages that must be compared with the results of a standardised measurement. The usefulness of this practice is questionable for the following reason. As it is shown in the present paper as well, modern power electronic converters emit low amount of harmonic current, and the effect caused by them is lesser than the effect of thousands of public consumers connecting to the same feeder. So even if a harmonic harms the limit of the standard, it is very likely to be the result of the communal consumers and not the distributed generator. For this reason, the idea of a future standard is starting to be outlined. This will regulate the harmonic voltage distortion of a generator alone - probably using $5 \%$ of the current limits. The wind generator under examination in this paper matches all requirements in force, and the mentioned coming one as well.

\section{Compensating the Voltage Change}

This part of the paper focuses on a problem that may be a challenge of the future. Challenge because sooner or later every electric power company will face it. This is the local voltage regulation. The traditional power electric system handles it a well known way that has to fulfil requirements of operation safety and thrift. The adjustable $\mathrm{HV} / \mathrm{MV}$ transformers do the crab of the process. The voltage of HV level is controlled by the system operators, their possibilities rely mainly on the reactive power producing capacity of the power stations. But this system has an advantage: the load curve of the communal consumers is well known and highly predictable, so apart from system malfunction the system operators can prepare well in advance. The penetration of distributed generation brings changes for this system, mostly of them the ones depending on hardly predictable energy sources - PV units and wind generators. Current paper focuses on the latter ones, partly because of their bigger incidence and the level of connection voltage. According to the valid market regulation, the electric power company in Hungary must buy all the energy produced on a renewable base - this means actually an active power acquisition. But most of the small generators are operated by companies independent from power electric companies, for whom system regulation is irrelevant. Because their profit is proportional to the injected active power, they try to avoid any reactive flow at the connection point, which ,philosophy” makes local voltage control more complicated. Certain steps must be made in the future to draw distributed generators into the control process. The authors examined a possible way of that. The explanation of the voltage rise or fall is simple: the current injected into the grid by the generator causes it. This voltage drop shall not exceed the $2 \%$ zone of the nominal value for MV grids, and the $3 \%$ zone for LV grids. A distributed generator that exceeds the limit must not be connected to the public grid. The further simulations will answer the question how big is this $2-3 \%$ limit. The formerly presented model have to be changed for these calculations, because one $800 \mathrm{~kW}$ generator rarely effects in harming the voltage limits, and otherwise it is less likely nowadays to build single turbines. For these reasons the generator was multiplied, resulting 5 parallel working units with total capacity of $4 \mathrm{MW}$. The distance between the wind park and the substation was investigated every $5 \mathrm{~km}$ between 5 and $30 \mathrm{~km}$. Such a wind park lies in a relative small area, so it can be set aside that production of individual generators differ.

The primary aim of the examination was to decide, whether the built-in power electronic converters can supply the reactive power, so these devices are connected to the $0,4 \mathrm{kV}$ side of the transformer. For the simulation, a script was written in the own programming language of PowerFactory called DPL. The algorithm runs the following way: the generator is connected to the grid with certain production, and a load-flow calculation is executed. The voltage of the connection point and the MV side of the substation is stored, and the amount of compensation is increased step by step. After definite number of calculations the voltage will reach the permitted limits; the reactive power and the loading of the transformer is stored this time. The cycle starts again after increase of the production. Of course the step parameters can be altered for a faster or for a stricter calculation.

\section{A. Connection of a Generator}

Figure 2. shows the expected results; the steady voltage rise becomes proportionately bigger as the distance between the substation and the connection point grows. A capacitive effect can be noticed when calculating longer distances - this will be later explained. The needed compensation highly depends on the length of the line. For example when connecting at the level of maximum power (4 MW) and using a $30 \mathrm{~km}$ line, the voltage reaches $23,799 \mathrm{kV}$. In contrast with this if we calculate with a $5 \mathrm{~km}$ length, voltage will not be higher than $22,344 \mathrm{kV}$, which is under the $2 \%$ limit of the standard, thus requires no compensation. The authors have marked 
a $1 \%$ limit as well for the following reason: if the electric power corporation allows the first connection to use up the $2 \%$ limit, it may happen that latter connections must be refused to keep the local voltage within the limit values. But if we only grant the first connection $1 \%$, this problem can be ignored in most situations. It must be mentioned, that the latter solution costs much more for the investor, but ensures more flexibility for the power company.

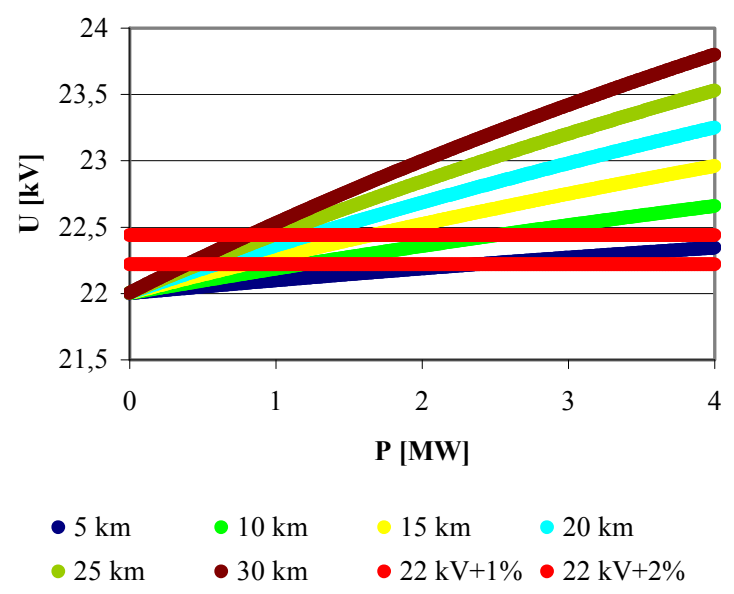

Fig. 2. Voltage rise of the $22 \mathrm{kV}$ side at the connection point of the generator

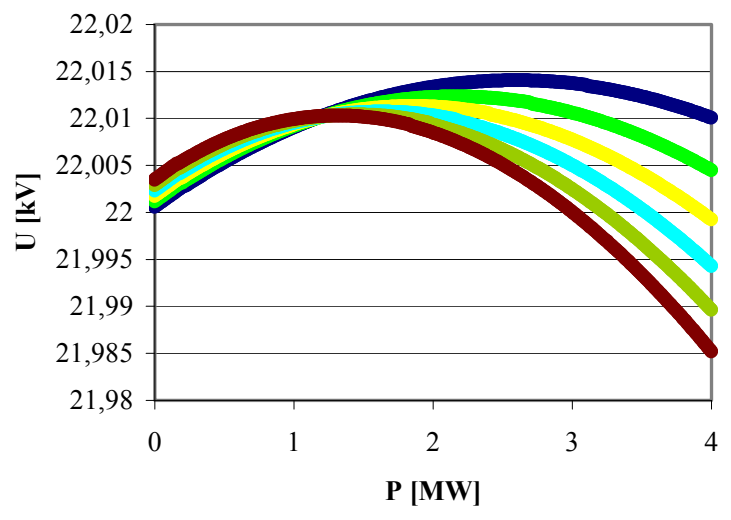

- $5 \mathrm{~km} \bullet 10 \mathrm{~km} \bullet 15 \mathrm{~km} \bullet 20 \mathrm{~km} \bullet 25 \mathrm{~km} \bullet 30 \mathrm{~km}$

Fig. 3. Voltage rise of the $22 \mathrm{kV}$ side at the substation

Figure 3. still presents the results of the first simulation, but in this case the voltage level of the substations MV side is shown. During simulations, the voltage level of the $120 / 22 \mathrm{kV}$ substation was held at nominal level on the $120 \mathrm{kV}$ side, and was free on the $22 \mathrm{kV}$ side. So for this, the results show the voltage drop of the transformer, and explain the formerly mentioned capacitive phenomenon. The active power where the characteristics cut belongs to the natural power of the line. Well known, that if the transported active power is below this amount, the line behaves as a reactive power generator, and if the transported active power is above the natural power, the line behaves as a reactive power consumer. If we count the theoretical natural power, the result will converge the result of the simulation well.

$$
P_{n a t}=\frac{U_{n}^{2}}{R_{0}}=\frac{U_{n}^{2}}{\sqrt{\frac{L^{\prime}}{C^{\prime}}}}=\frac{(22 \mathrm{kV})^{2}}{\sqrt{\frac{1,2732 \mathrm{mH} / \mathrm{km}}{8 n F / k m}}} \cong 1,2132 \mathrm{MW}
$$

This phenomenon must be taken into consideration, because it affects the amount of the necessary local compensation, especially with longer distribution lines.

\section{B. Disconnection of a Generator}

Going on the authors will now focus on the phenomena during disconnection process. The basics of the simulation don't change: the investigated system is in steady state, the generator produces only active power, and the voltage at the connection point is at nominal level. Disconnecting the generator will result now in a voltage fall. The algorithm used this time is slightly different, because before disconnection, the voltage level must be compensated, and only thereafter can the simulation run in the way it was formerly explained.

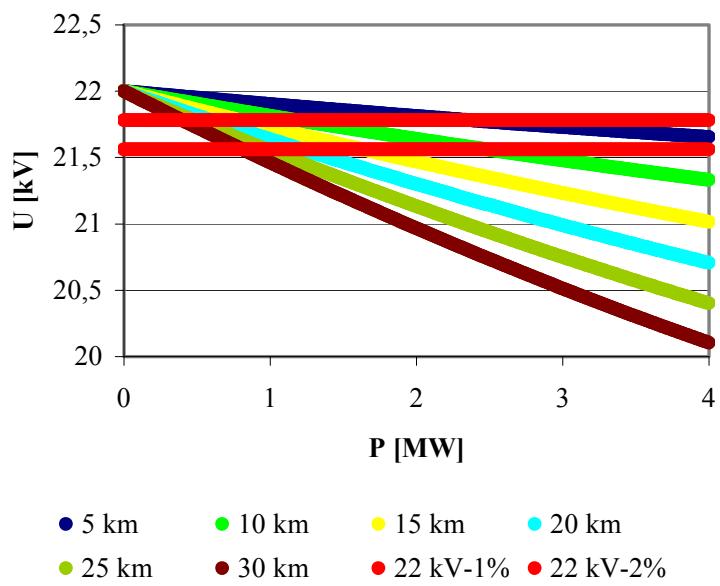

Fig. 4. Voltage fall of the $22 \mathrm{kV}$ side at the connection point of the generator

The characteristics seen on Figure 4. look very similar to the ones on Figure 2., as it was expected, because the process is the same in general. In this case when the length of the power line is $30 \mathrm{~km}$, the voltage falls to $20,104 \mathrm{kV}$, that is $8,61 \%$ lower than the nominal value (for the same conditions the simulation of connection resulted $8,18 \%$ difference). When the length is decreased to $5 \mathrm{~km}$, the voltage of the connection point is $21,654 \mathrm{kV}$, this equals $1,57 \%$ fall ( $1,56 \%$ for the case of connection). As we can see there is almost no numeral difference between the effects of connection and disconnection.

Figure 5. displays substation voltages again, this time after the disconnection of the generator. The difference is now significant between the case of connection and disconnection; the voltage change is way bigger, but the capacitive effect has almost no impact on the results. Of course in real life things would look somewhat different, 
because in the present model the generator is connected to the substation directly, and all consumers connected to this feeder are ignored.

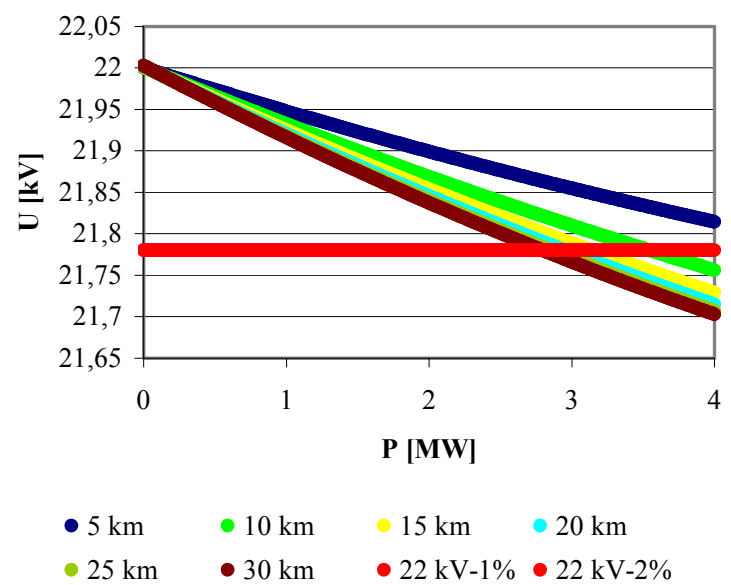

Fig. 5. Voltage fall of the $22 \mathrm{kV}$ side at the substation

\section{Compensating the Voltage Change}

Well-known methods are available for reactive power compensation like use of shunt capacitors or reactors. But high percent of the modern distributed generators have modern built-in power electronic converters, which are capable of producing and consuming certain amount of reactive power. The investigated wind generator also has such devices; the product of Enercon GmbH. suits the generation in parameters well. Using the P-Q characteristics if the converter - which was available - it can be decided, whether they can supply the requested compensation or not. So now we are focusing on local compensation that is placed on the LV side of the transformer. Let's see the outcome!

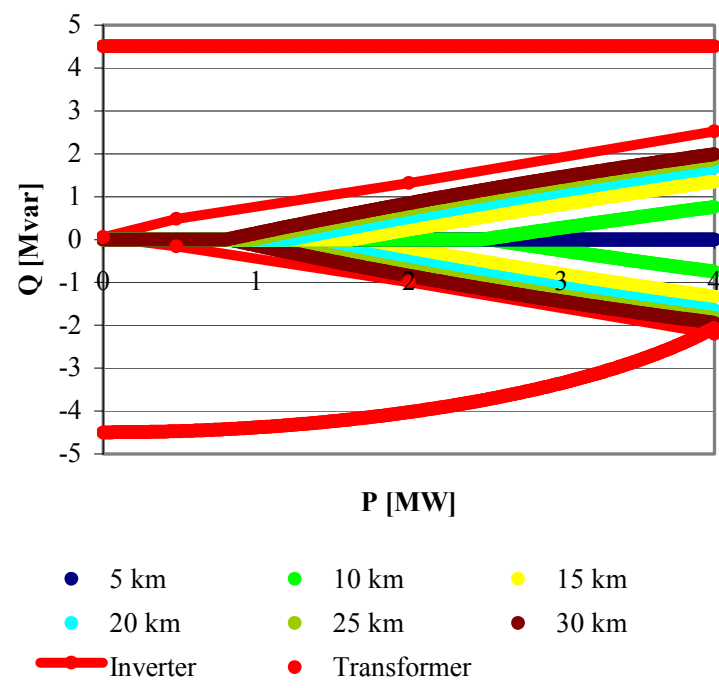

Fig. 6. Compensation needed to keep the $2 \%$ limit

Figure 6. summarizes everything that has been mentioned up to this point. There are two limits displayed: one for the inverter and one for the transformer. The inverter limit stands for the formerly mentioned P-Q characteristic of the power electronics. It shows, that the compensation capacity is not symmetrical: it can consume reactive power up to 2,2 Mvar, but it can produce as much as 2,52 Mvar - all of these values should be read as the aggregation for 5 parallel generators of course. Considering the power factor this means $\cos \varphi=0,876$ inductive and $\cos \varphi=0,846$ capacitive values, that are better for our purpose than the permitted limit of the generator alone. Because of the asymmetrical characteristics of the inverter, the needed compensation after connection - this means consumption of reactive power - is narrowly within limits, while when dealing with disconnection, the potential is enough for a much longer power line. The real point in Figure 6 . is that it obviously proves that the built-in devices are suffice to deal with the compensation.

The other of the two limits is the transformer. We decided to include this in the model as well, because the manufacturing company probably delivers the same type of transformer for a certain type of wind turbine, and it can happen, that the apparent power that is to be injected into the grid is bigger than the apparent power of the transformer. But both limits can be ignored when compensation is made to meet the $2 \%$ limit compared to the nominal voltage level. We can observe though else, if we'd like to keep the voltage between the $1 \%$ limits, as Figure 7 shows.

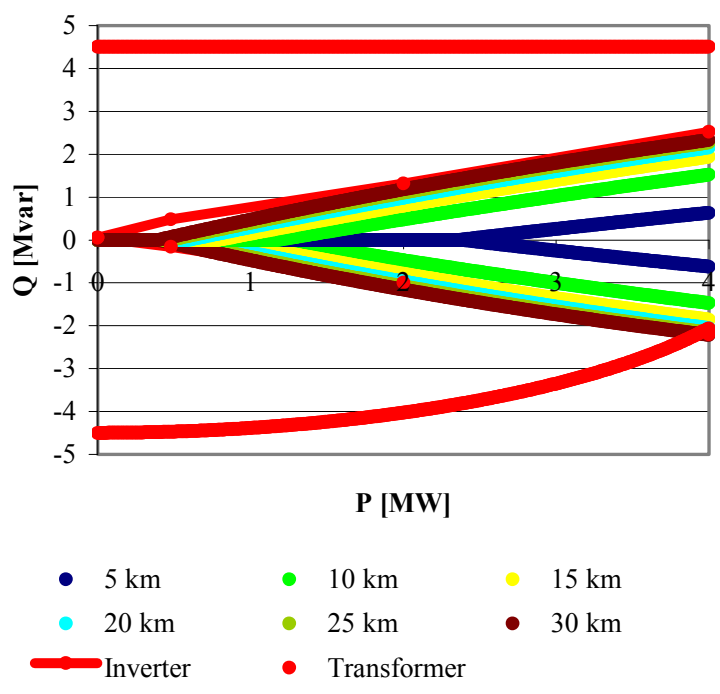

Fig. 7. Compensation needed to keep the $1 \%$ limit

If the electric power company wants to use the principle mentioned before - namely to „leave space” for the latter connections - the possibilities are waning. After connection of a generator we can compensate the needed reactive power for all power line length up to $20 \mathrm{~km}$, but if the feeder is longer than that, only situations with low generation can be treated. In spite all of this, after disconnection the power electronics can produce sufficient reactive power for all cases investigated. A difference for the $2 \%$ case is that the transformer limit cuts some of the curves. The reason behind this is that 
though the power of the generator is $800 \mathrm{~kW}$, the apparent power of the transformer is just $900 \mathrm{kVA}$, so if generation is at maximum level, the transformer already operates with the loading of $87 \%$. This problem only occurs when the power line is longer than $20 \mathrm{~km}$ and the wind generators are delivering nearly maximum power. A transformer can operate though for certain time in overload without damage. Assuming that the generation will rarely reach maximum level - in Hungary utilization level of wind generators is low - this overload can be permitted. Even if we would like to avoid this for some reasons, we still have two choices; we can be satisfied to compensate in this cases in view of the standard's $2 \%$ limit, or we can order to compensate as much as the transformer limit allows, and the voltage will be between 1 and $2 \%$ compared to nominal value.

\section{Constructional changes}

As seen, the built-in power electronics are capable of supplying the reactive power compensation we demand. We cannot leave out of consideration though, that minor constructional modifications must be made. These of course mean reasonable extra cost compared to the whole

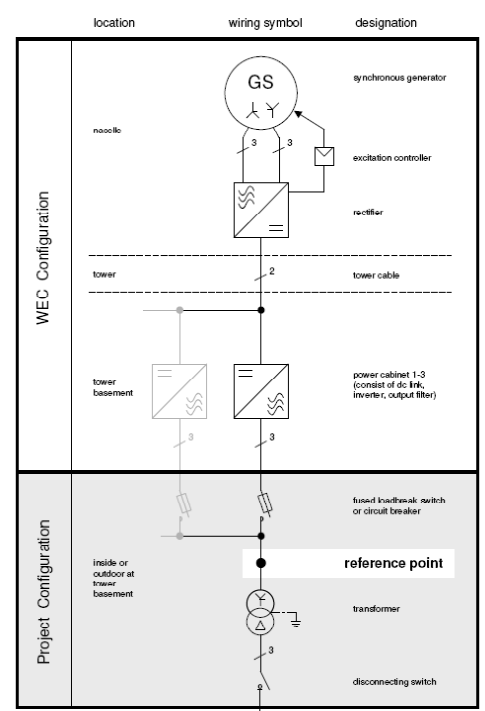

Fig. 8. Electric scheme of the wind generator project, if the procurer ask for them in order. Figure 8. shows the electric scheme of the wind generator. The marked „reference point" is the point where the P-Q characteristic is defined. The main problem in our respect is that the load break switches are located between the converters and the grid. Therefore if we would like to prevent the local voltage from falling after the disconnection of the unit, the device that should supply reactive power is also disconnected. So it would be practical to change the structure so, that the load break switches are placed between the inverter and the grid.

This solution can be compared to the placing of a static compensator. Set aside on which voltage levels is such a device available on the market, because it can connect to MV level at the grid connection point, or with a tertiary winding to the transformer - the point is that it should work properly. Such a compensator costs about 40-60 Euros/kVA. In our model the highest reactive power needed was 2,328 Mvar, that matches a device costing
140000 Euros! This sum cannot be disregarded, so the authors conclude, that it is worth to order the construction modifications in advance.

\section{Conclusion and Future Work}

Present paper focuses on two very actual questions concerning the future of power electric systems. Market courses predict, that the number of distributed generators in service will steadily grow. Harmonic source identification concluded, that the fears around the harmonic injection of power electronic converters used in distributed generators exaggerated. Though the method of monoparameter variation could not be used because of the inadequate measurement registration file, the analysis of the 10 minute averages showed results. The authors conclude, that the examined wind generator is not a dominant harmonic source, and fulfils the limits of the MSZ EN 50160 Standard concerning the connection terms.

Second part of the paper focused on the steady voltage change caused by changing generation, which is based on the requirements of the MSZ EN 50160 Standard as well. Concerning MV level, this must be smaller than $2 \%$ of the nominal value. To investigate this problem, a computer model was created with PowerFactory. Simulations showed, that this limit could only be kept to with the use of some kind of local compensation. The authors have chosen to use the built-in power electronics of the generator. Results show, that this solution can be sufficient to keep the voltage between the $2 \%$ limits. Even if the electric power company orders the owner of the generator to keep to the $1 \%$ limit the potential of the converters proves to be satisfactory in most of the situations. The required constructional modifications can also be made with little costs.

Future work is determined by the market liberalisation process. After January 2008 in Hungary anyone can connect a micro generator to the communal grid with power up to $8 \mathrm{~kW}$ (some companies prefer $50 \mathrm{~kW}$ ). The authors believe, that the number of such generators will increase fast, and so will their effect on line power. There is no regulation in force to draw these units into local voltage control, but the forthcoming years must bring possible solutions. This sets the target for future work.

\section{References}

[1] A. Dán, T. Tersztyánszky, Gy. Varjú, „Electric Power Quality" (Villamosenergia minöség), InvestMarketing Ltd., Budapest, Hungary, 2006.

[2] MSZ EN Standard 50160, Voltage characteristics of electricity supplied by public distribution systems, 2001

[3] ***, „DIgSILENT PowerFactory Version 13.1 Manual", nov. 2005 